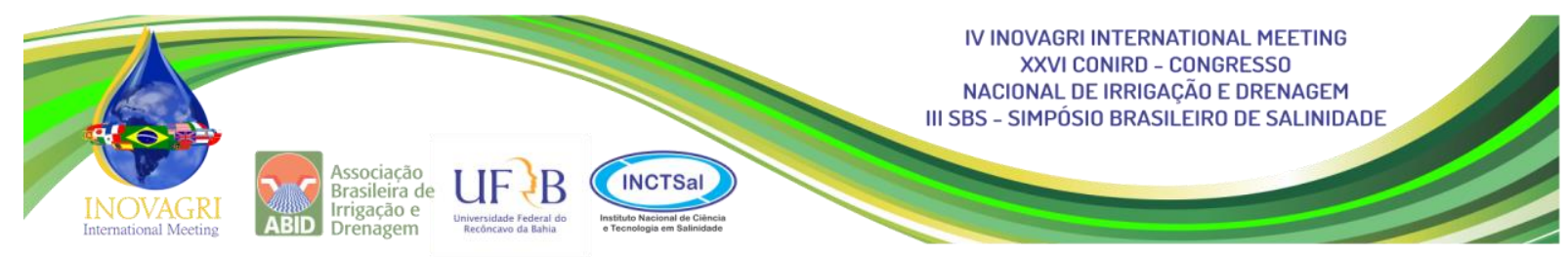

\title{
AVALIAÇÃO DE LINHAGENS DE FEIJOEIRO-COMUM ADAPTADOS AO VALE DO SUBMÉDIO SÃO FRANCISCO
}

\author{
M. Calgaro ${ }^{1}$, L. C. Mello ${ }^{2}$, L. C. de Faria ${ }^{3}$, W. L. Simões ${ }^{4}$, J. M Pinto ${ }^{5}$, M. Alves ${ }^{6}$
}

RESUMO: Neste trabalho avaliaram-se linhagens de feijoeiro-comum do tipo carioca de ciclo normal, para a determinação do potencial agronômico de linhagens elite que se adaptem aos diferentes sistemas de produção no Vale do Submédio São Francisco. O delineamento experimental utilizado foi o de blocos completamente casualizados, onde cada unidade experimental foi constituída de três repetições e parcelas de quatro linhas de quatro metros de comprimento. O estudo faz parte de um projeto da Embrapa Arroz e Feijão e tem como parceira a Embrapa Semiárido no desenvolvimento das avaliações de campo. Os materiais foram irrigados por gotejamento superficial, com base na $\mathrm{ET}_{0}$ determinada por meio de uma estação agrometeorológica. Foram realizadas avaliações de características agronômicas de importância, como a produção e a produtividade de grãos, ambas medidas em kg.ha ${ }^{-1}$. Os resultados obtidos mostram materiais com potencial próximo a variedades consagradas no mercado brasileiro e utilizadas como testemunha como é o caso da linhagem CNFC 16876, que produziu 2.690,75 kg.ha-1 ${ }^{-1}$ o que significa uma diferença de apenas $1,36 \%$ a menos que a variedade testemunha PÉROLA, que produziu $2.727,78 \mathrm{~kg} \cdot \mathrm{ha}^{-1}$.

PALAVRAS-CHAVE: Irrigação, melhoramento genético, Phaseolus vulgaris.

\section{EVALUATION OF COMMON BEAN LINES ADAPTED TO THE SUB MIDLE SÃO FRANCISCO VALLEY}

SUMMARY: In this work, common bean lines of the normal-cycle type of bean were evaluated for the determination of the agronomic potential of elite lineages adapted to the different production systems in the sub midle São Francisco Valley. The experimental design was completely randomized blocks, where each experimental unit consisted of three replicates and plots of four lines of four meters in length. The study is part of an Embrapa Arroz e

\footnotetext{
${ }^{1}$ Doutor, Pesquisador da Embrapa Semiárido, Petrolina - Pernambuco. Email: marcelo.calgaro@embrapa.br

${ }^{2}$ Doutor, Pesquisador da Embrapa Arroz e Feijão, Santo Antônio de Jesus - Goias. Email: leonardo.mello@embrapa.br

${ }^{3}$ Doutor, Pesquisador da Embrapa Arroz e Feijão, Santo Antônio de Jesus - Goias. Email: luis.faria@embrapa.br

${ }^{4}$ Doutor, Pesquisador da Embrapa Semiárido, Petrolina - Pernambuco. Email: welson.simoes@embrapa.br

${ }^{5}$ Doutor, Pesquisador da Embrapa Semiárido, Petrolina - Pernambuco. Email: jose-maria.pinto@embrapa.br

${ }^{6}$ Bolsista, Acadêmico de Ciências Biológicas da UPE, Petrolina - Pernambuco. Email: manoelalves2013@ hotmail.com
} 
Feijão project and is partnered with Embrapa Semiarido in the development of field evaluations. The materials were irrigated by surface dripping, based on $\mathrm{ET}_{0}$ determined by means of an agrometeorological station. Evaluations of important agronomic characteristics,

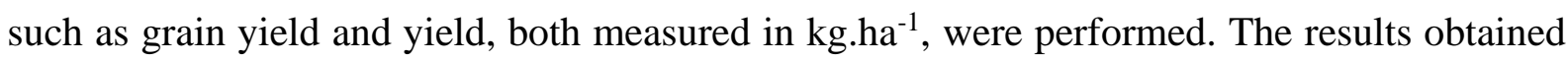
show materials with potential close to varieties consecrated in the Brazilian market and used as a witness, as is the case of line CNFC 16876, which produced 2,690.75 kg.ha ${ }^{-1}$, which means a difference of only $1.36 \%$ a Less than the pearling variety, which produced $2,727.78$ $\mathrm{kg} \mathrm{ha}^{-1}$.

KEYWORDS: Irrigation, genetical enhancement, Phaseolus vulgaris

\section{INTRODUÇÃO}

O cultivo de feijoeiro é bastante difundido em todo o território nacional, sendo o mesmo ainda reconhecido como cultura de subsistência em pequenas propriedades, muito embora tenha havido, nos últimos 25 anos, crescente interesse de produtores de outras classes, com adoção de tecnologias avançadas, incluindo irrigação, manejo fitossanitário e colheita mecanizada (Barbosa \& Gonzaga, 2012). Cerca de 90\% dos empreendimentos agrícolas produtores de feijão no Brasil são considerados como agricultura familiar segundo a classificação utilizada pelo IBGE no Censo Agropecuário 2006, baseada na Lei 11.326, de 24 de julho de 2006 (BRASIL, 2006).

O Brasil Além de ser o maior produtor mundial, também é o maior consumidor de feijão, sendo que, a preferência tradicional de consumo tem sido por grãos do tipo comercial carioca, o que justifica a intensidade de esforços no sentido de desenvolver linhagens superiores com esse tipo de grão, que associam o maior número possível de características que expressem o fenótipo desejável (Del Peloso et al., 2003).

O uso de técnicas de irrigação e adubação adequadas, acompanhados de uma população de plantas adequada, permitem alcançar maiores níveis de produtividade do feijoeiro-comum, com rendimentos superiores a $3.000 \mathrm{~kg} \cdot \mathrm{ha}^{-1}$, compatíveis com uma agricultura irrigada de bases racionais (Azevedo et al., 2008). No Vale do São Francisco os produtores têm obtido produtividades entre de 1.500 a $2.000 \mathrm{~kg} \cdot \mathrm{ha}^{-1}$, segundo Costa \& Lopes, (1999) com o uso de técnicas de irrigação. 
O objetivo deste trabalho foi o de avaliar linhagens de feijão carioca de ciclo normal, para determinação do potencial agronômico de linhagens elite que se adaptem aos diferentes sistemas de produção na região Nordeste conduzidas sob irrigação por gotejamento.

\section{MATERIAL E MÉTODOS}

A pesquisa foi conduzida no Campo Experimental de Bebedouro, pertencente a Embrapa Semiárido, localizado no Município de Petrolina, PE, (latitude: $9^{\circ} 09^{`} \mathrm{~S}$, longitude: $40^{\circ} 22^{\prime} \mathrm{W}$, altitude: $365,5 \mathrm{~m}$ ), no período seco e chuvoso (maio a outubro). O solo do local segundo EMBRAPA, (2006) é classificado como Latossolo vermelho amarelo distrófico. A classificação climática, segundo Köppen, (1918) é do tipo BSWh, ou seja, tropical semiárido, conforme descrito em Reddy \& Amorim Neto (1983). As chuvas concentram-se entre os meses de novembro e abril, com precipitação média anual em torno de $426 \mathrm{~mm}$, irregularmente distribuídas. A temperatura média anual é de $28,3^{\circ} \mathrm{C}$, variando entre 21 e $32^{\circ} \mathrm{C}$, com uma evaporação média anual em torno de $2000 \mathrm{~mm}$, umidade relativa do ar média anual de 68,5\%, 3000 horas anuais de brilho solar e velocidade média do vento de 2,3 m.s ${ }^{-1}$ (EMBRAPA, 2016).

Foram avaliados 15 genótipos de feijão-comum do tipo carioca de ciclo normal, distribuídos em blocos inteiramente casualizados, com três repetições, sendo que a semeadura foi realizada no dia 22 de junho de 2016, em parcelas de 4 fileiras com 4 metros de comprimento, espaçadas $0,3 \mathrm{~m}$ entre fileiras. A adubação foi realizada conforme recomendação técnica para o cultivo na região, com aplicação de $20 \mathrm{~kg} \cdot \mathrm{ha}^{-1}$ de N, $20 \mathrm{~kg} \cdot \mathrm{ha}^{-1}$ de $\mathrm{P}_{2} \mathrm{O}_{5}$ e $20 \mathrm{~kg}$.ha- ${ }^{-1}$ de $\mathrm{K}_{2} \mathrm{O}$. A adubação de cobertura foi aplicada $40 \mathrm{~kg}$.ha-1 de $\mathrm{N}$, aos 20 dias após a emergência. Os tratos culturais realizados foram os recomendados para a cultura do feijoeiro, segundo Araújo et al., (1996), sendo que no controle de plantas daninhas foi efetuado capina manual realizada a cada 20 dias.

Para proporcionar pleno desenvolvimento a cultura foi utilizado o método de irrigação localizada, a fim de prover a condição adequada de desenvolvimento, uma vez que o período de cultivo ocorreu na época onde as precipitações são ausentes. O sistema utilizado foi de gotejamento superficial, com gotejadores espaçados $0,5 \mathrm{~m}$ entre si e com vazão média de 2,0 L.h ${ }^{-1}$.

O manejo da irrigação foi realizado com base na evapotranspiração da cultura (Allen et al., 1998), sendo que após a semeadura a umidade do solo foi elevada à capacidade de campo 
e daí por diante as irrigações foram realizadas, visando repor a quantidade de água evapotranspirada pela cultura, com turno de rega de dois dias. O coeficiente de cultivo utilizado foi o sugerido por Silveira \& Stone, (2007), sendo os valores utilizados de coeficientes de cultura $(\mathrm{Kc})$ em cada fase da cultura estão descritos na Tabela 1. Foi realizado um teste para determinar o coeficiente de uniformidade de distribuição de água (CUD), segundo método sugerido por Keller \& Karmeli (1975), o qual resultou em um CUD de 96\%, o que na classificação apresentada por Merriam \& Keller (1978) é considerado como sendo excelente.

A colheita foi realizada no dia 19 de setembro de 2016, colhendo-se as duas fileiras centrais de 4 metros de comprimento, totalizando uma área útil colhida de $2,4 \mathrm{~m}^{2}$. A área colhida, no momento da maturação completa, foi equivalente a 2,4 $\mathrm{m}^{2}$ correspondendo as duas fileiras centrais de cada repetição. Foram avaliadas a produção total e a produtividade média em kg.ha¹. As médias das produtividades estimadas, foram comparadas pelo teste de Scott-Knott a $5 \%$ de probabilidade.

\section{RESULTADOS E DISCUSSÃO}

Os resultados das produtividades de grãos encontradas são apresentados na Tabela 2. O rendimento médio de grãos do experimento foi de 2.112,46 kg.ha-1, com coeficiente de variação (CV) de 13,59\%, o que segundo Moraes et al., (2007) é classificado como um pouco elevado, mas ainda dentro do máximo tolerado que é de 20\%. O ciclo médio dos genótipos no ensaio foi de 86 dias. O maior rendimento de grãos, em valor absoluto observado, foi o da testemunha cultivar Pérola $\left(2.727,78 \mathrm{~kg} \cdot \mathrm{ha}^{-1}\right)$, enquanto que a melhor linhagem, CNFC 16876, obteve um rendimento médio de 2.690,75 kg.ha-1, não diferindo estatisticamente da cultivar PÉROLA, e produzindo apenas $1,36 \%$ a menos em relação a esta, o que estatisticamente não o diferenciou da testemunha. Ainda entre as linhagens de desempenho superior, tivemos a CNFC 15805, CNFC 16902 e CNFC 15853, todas com produtividades estatisticamente semelhantes a testemunha e com o percentual de redução de produtividade em relação a testemunha, inferior a $10 \%$, evidenciando o seu alto potencial de cultivo na região do Vale do Submédio São Francisco.

O genótipo CNFC 15826, embora tenha apresentado uma produtividade média de $2.284,22 \mathrm{~kg} \cdot \mathrm{ha}^{-1}$ o que não o diferencia estatisticamente da testemunha, teve uma redução de $16,26 \%$ com relação a esta, percentual este que se apresenta mais próximo aos materiais que apresentaram um menor desempenho. 
Dentre os genótipos que apresentaram as menores produtividades, estão 10 materiais cujas produtividades oscilaram entre $1.544,83 \mathrm{~kg} \cdot \mathrm{ha}^{-1} \mathrm{e} 2.205,21 \mathrm{~kg} \cdot \mathrm{ha}^{-1}$ em valores absolutos, o que não os tornam menos interessantes para futuros cruzamentos ou testes. $\mathrm{O}$ menor rendimento de grãos foi o do genótipo CNFC 15859 com uma produtividade média de $1.544,83 \mathrm{~kg} \cdot \mathrm{ha}^{-1}$.

\section{CONCLUSÕES}

Nenhum genótipo superou estatisticamente a cultivar PÉROLA. Dentre os genótipos estudados, o que apresentou o maior rendimento de grãos em valores absolutos foi CNFC 16876, com uma produtividade de 2.690,75 kg.ha-1 . Destacaram-se ainda os genótipos CNFC 15805, CNFC 16902, CNFC 15853, CNFC 15826, todos com produtividades estatisticamente semelhantes a testemunha, evidenciando seu potencial para cultivo na região do Vale do Submédio São Francisco.

\section{AGRADECIMENTOS}

Aos funcionários do Campo Experimental de Bebedouro e aos estagiários e bolsistas que tornaram possível a realização desse trabalho.

\section{REFERÊNCIAS BIBLIOGRÁFICAS}

ALLEN, R. G.; PEREIRA, L. S.; RAES, D.; SMITH, M. Crop evapotranspiration: Guidelines for computing crop water requirements. Rome: FAO, 1998. 300 p. (FAO - Irrigation and Drainage Paper, 56).

ARAÚJO, Ricardo S.; RAVA, Carlos A.; STONE, Luis F.; ZIMMERMANN, Maria J. O. Cultura do Feijoeiro Comum no Brasil. Piracicaba: Associação Brasileira para Pesquisa da Potassa e do Fosfato, 1996.

AZEVEDO, J. A. de; SILVA, E. M. da; RODRIGUES, G. C.; GOMES, A. C. Produtividade do feijão de inverno influenciada por irrigação, densidade de plantio e adubação em solo de Cerrado. Planaltina, DF: Embrapa Cerrados, 2008. 3 p. (Embrapa Cerrados. Comunicado técnico, 145). 
BARBOSA, F. R.; GONZAGA, A. C. de O. (Ed.). Informações técnicas para o cultivo do feijoeiro-comum na Região Central-Brasileira: 2012-2014. Santo Antônio de Goiás: Embrapa Arroz e Feijão, 2012. 247 p. (Embrapa Arroz e Feijão. Documentos, 272).

BRASIL. Presidência da República. Lei 11.326, de 24 de julho de 2006. Disponível em: <http://www. planalto.gov.br/ccivil_03/_ato2004-2006/2006/lei/111326.htm>. Acesso em 10 out. 2012.

COSTA, A.F. da; LOPES, L. H. de O. Recursos geneticos e melhoramento do feijoeiro comum em Pernambuco. In: QUEIROZ, M. A. de; GOEDERT, C. O.; RAMOS, S. R. R. (Ed.). Recursos genéticos e melhoramento de plantas para o Nordeste brasileiro. Petrolina: Embrapa Semi-Árido; Brasília, DF: Embrapa Recursos Genéticos e Biotecnologia, 1999.

DEL PElOSO, M. J.; MELO, L. C.; FARIA, L. C. de; COSTA, J. G. C. da; RAVA, C. A.; CARNEIRO, G. E. de S.; SOARES, D. M.; CABRERA DÍAZ, J. L.; ABREU, Â. de F. B.; FARIA, J. C. de; SARTORATO, A.; SILVA, H. T. da; BASSINELlO, P. Z.; ZIMMERMANN, F. J. P. BRS Pontal: nova cultivar de feijoeiro comum de tipo de grão carioca com alto potencial produtivo. Santo Antônio de Goiás: Embrapa Arroz e Feijão, 2003. 2 p. (Embrapa Arroz e Feijão. Comunicado técnico, 64).

EMBRAPA. Centro Nacional de Pesquisa de Solos. Sistema Brasileiro de Classificação de Solos. 2 ed. Rio de Janeiro: Embrapa Solos, 2006.

KELLER, J.; KARMELI, D. Trickle irrigation desing. Glendora: Rain Bird Sprinkler Manufacturing, 1975.

KÖPPEN, W. Klassification der klimate nach temperatur, niederschlag und jahreslauf. Petermanns Geographische Mitteilungen, Gotha, v. 64, p. 193- 203, 1918.

MERRIAM, J.L.; KELLER, J. Farm irrigation system evaluation: a guide for management. Logan: Utah State University, 1978.

MORAES, R. M. A. de; IGNACZAK, J. C.; DEL PELOSO, M. J.; FARIA, L. C. de; MELO, L. C. Ensaio de valor de cultivo e uso de feijão carioca em Coxilha (RS), Embrapa Trigo, safra e safrinha, 2006/07. Passo Fundo: Embrapa Trigo, 2007. 10 p. html. (Embrapa Trigo. Circular técnica online, 22).

SILVEIRA, P; M da; STONE, L; F. Manejo de irrigação. IN: Agencia de Informação Embrapa Feijão. Disponível em: 
http://www.agencia.cnptia.embrapa.br/Agencia4/AG01/arvore/AG01_86_1311200215104.ht ml. Acesso em 13 de novembro 2016.

SCOTT, A. J.; KNOTT, M. A cluster analysis method for grouping means in the analysis of variance. Biometrics, Washington, v. 30, n.2 p.507-512, 1974.

Tabela 1. Coeficiente de cultura $(\mathrm{Kc})$ para as três principais fases do ciclo da cultura do feijão-comum.

\begin{tabular}{lcc}
\hline \multicolumn{1}{c}{ Fases da cultura } & $\begin{array}{c}\text { Duração da fase } \\
\text { (dias) }\end{array}$ & Kc \\
\hline Germinação - Início da floração & 35 & 0,69 \\
\hline Floração - Início do desenvolvimento de vagens & 25 & 1,28 \\
\hline Desenvolvimento de vagens - Maturação & 20 & 1,04 \\
\hline Fonte: Silveira e Stone, 2016
\end{tabular}

Fonte: Silveira e Stone, 2016.

Tabela 2. Produtividades médias em kg.ha-1.

\begin{tabular}{|c|c|}
\hline Nome & $\begin{array}{c}\text { Produtividade média } \\
\left(\mathrm{kg}^{\mathrm{h}} \mathrm{ha}^{-1}\right)\end{array}$ \\
\hline PÉROLA* & $2.727,78 \quad a$ \\
\hline CNFC 16876 & $2.690,75 \mathrm{a}$ \\
\hline CNFC 15805 & $2.601,57 \quad \mathrm{a}$ \\
\hline CNFC 16902 & $2.475,50 \quad \mathrm{a}$ \\
\hline CNFC 15853 & $2.456,11$ a \\
\hline CNFC 15826 & $2.284,22$ a \\
\hline CNFC 15839 & $2.205,21 \quad b$ \\
\hline CNFC 15850 & $2.146,36 \mathrm{~b}$ \\
\hline CNFC 15713 & $2.143,46 \quad b$ \\
\hline CNFC 15820 & $2.059,85 \mathrm{~b}$ \\
\hline CNFC 15743 & $2.039,88 \mathrm{~b}$ \\
\hline CNFC 15801 & $1.988,76 \mathrm{~b}$ \\
\hline CNFC 15854 & $1.861,63 \mathrm{~b}$ \\
\hline CNFC 15860 & $1.733,65 \mathrm{~b}$ \\
\hline CNFC 16709 & $1.727,51 \quad b$ \\
\hline CNFC 15859 & $1.544,83 \mathrm{~b}$ \\
\hline
\end{tabular}

Médias seguidas da mesma letra não diferem entre si pelo teste de Scott-Knott a 5\% de probabilidade. ${ }^{*}$ Testemunha. 